\title{
Luteolin Regulates the Differentiation of Regulatory $T$ Cells and Activates IL-10-Dependent Macrophage Polarization against Acute Lung Injury
}

\author{
Ke Xie, Yu-sen Chai, Shi-hui Lin $\mathbb{D}$, Fang Xu $\mathbb{D}$, and Chuan-jiang Wang $\mathbb{D}$ \\ Department of Critical Care Medicine, The First Affiliated Hospital of Chongqing Medical University, Chongqing, China \\ Correspondence should be addressed to Fang Xu; xufang828@126.com and Chuan-jiang Wang; wangchuanjiang@cqmu.edu.cn
}

Received 13 September 2020; Revised 8 December 2020; Accepted 22 December 2020; Published 18 January 2021

Academic Editor: Youmin Kang

Copyright (C) $2021 \mathrm{Ke}$ Xie et al. This is an open access article distributed under the Creative Commons Attribution License, which permits unrestricted use, distribution, and reproduction in any medium, provided the original work is properly cited.

\begin{abstract}
Objectives. Inflammatory disease characterized by clinical destructive respiratory disorder is called acute lung injury/acute respiratory distress syndrome (ALI/ARDS). Studies have shown that luteolin exerts anti-inflammatory effects by increasing regulatory $\mathrm{T}$ cells (Tregs). In this study, we aimed to determine the effects of luteolin on ALI/ARDS and Treg differentiation. Methods. In this paper, we used cecal ligation puncture (CLP) to generate an ALI mouse model to determine the effects of luteolin on ALI/ARDS. Lung tissues were stained for interleukin- (IL-) 17A and myeloperoxidase (MPO) by immunohistochemical analysis. The levels of Treg-related cytokines in serum and bronchoalveolar lavage fluid (BALF) of mice were detected. The protein levels of NF- $\kappa \mathrm{B}$ p65 in lung tissues were measured. Macrophage phenotypes in lung tissues were measured using immunofluorescence. The proportion of Tregs in splenic mononuclear cells and peripheral blood mononuclear cells (PBMCs) was quantified. Furthermore, in vitro, we evaluated the effects of luteolin on Treg differentiation, and the effects of IL-10 immune regulation on macrophage polarization were examined. Results. Luteolin alleviated lung injury and suppressed uncontrolled inflammation and downregulated IL-17A, MPO, and NF- $\kappa \mathrm{B}$ in the lungs of CLP-induced mouse models. At this time, luteolin upregulated the level of IL- 10 in serum and BALF and the frequency of $\mathrm{CD} 4{ }^{+} \mathrm{CD} 25^{+} \mathrm{FOXP} 3^{+}$Tregs in PBMCs and splenic mononuclear cells of CLP mice. Luteolin treatment decreased the proportion of M1 macrophages and increased the proportion of M2 macrophages in lungs of CLP-induced mouse models. In vitro, administration of luteolin significantly induced Treg differentiation, and IL-10 promoted the polarization of M2 macrophages but reduced the polarization of M1 macrophages. Conclusions. Luteolin alleviated lung injury and suppressed uncontrolled inflammation by inducing the differentiation of $\mathrm{CD}^{+} \mathrm{CD} 25^{+} \mathrm{FOXP}^{+}$Tregs and upregulating the expression of IL-10. Furthermore, the anti-inflammatory cytokine IL-10 promoted polarization of M2 macrophages in vitro. Luteolin-induced Treg differentiation from naïve CD4 $4^{+} \mathrm{T}$ cells may be a potential mechanism for regulating IL-10 production.
\end{abstract}

\section{Introduction}

Acute respiratory distress syndrome (ARDS) is a clinically devastating respiratory disorder $[1,2]$. Even though clinical management and treatment have improved recently, the mortality rate of severe ARDS remains approximately $46.0 \%$ [3]. One of the most promising pharmacological approaches for treating ARDS is based on the pathophysiology of ARDS, i.e., the injury of alveolar epithelial and endothelial cells. However, studies have shown that molecular mechanisms underlying the inflammatory responses are critical to the development of ARDS. In the control and treat- ment of diseases, it is necessary to adjust and control immune cells and lung microenvironment in a timely manner [4]. Current research studies focus on the identification of drugs for the treatment of ARDS. Personalized pharmacologic therapy for ARDS is one of the most promising ways to treat this disorder [5].

Luteolin (3,4,5,7-tetrahydroxyflavone) can be extracted from many medicinal plants and some common vegetables and fruits, including broccoli, onion leaves, carrot, pepper, cabbage, and apple $[6,7]$. It possesses numerous beneficial medicinal properties, such as antioxidative, anti-inflammatory, cardioprotective, neuroprotective, and anti-allergic $[8$, 
9]. A previous study on acute lung injury (ALI) caused by sepsis has shown that luteolin has protective therapeutic effects [10]. Regulatory T cells (Tregs) are special $\mathrm{T}$ cells that have anti-inflammatory and antiapoptotic properties. These cells can reduce inflammation-induced tissue damage and create an appropriate immune microenvironment via multiple mechanisms. In ARDS, Tregs function as a metronome for "inflammation factor storm" regulation [4]. They can serve as targets for the treatment of ARDS. Tregs are generated by the differentiation of naïve $\mathrm{CD} 4^{+} \mathrm{T}$ cells. These cells exert their immunoregulatory effects by affecting macrophage polarization through interleukin-10 (IL-10) [11, 12]. Interestingly, luteolin alleviates airway inflammation mainly because it promotes the differentiation of $\mathrm{CD} 4^{+} \mathrm{CD} 25^{-} \mathrm{T}$ cells into $\mathrm{CD} 4^{+} \mathrm{CD} 25^{+}$Tregs [13]. In the present study, we investigated whether luteolin exerts a therapeutic effect through regulation of Tregs and IL-10 immune modulation in ARDS.

To validate our hypothesis, we establish a cecal ligation and puncture- (CLP-) induced ALI mouse model and measured the proportion of Tregs in splenic mononuclear cells and peripheral blood mononuclear cells (PBMCs), and identified the polarization of macrophages in lung tissue. Furthermore, in vitro, we evaluated the effects of luteolin on Treg differentiation and the effects of IL-10 immune regulation on macrophage polarization.

\section{Materials and Methods}

2.1. Animals. All the mice in this experiment were male C57BL/6 mice aged 8-12 weeks and were purchased from the Experimental Animal Center of Chongqing Medical University. Before the experiment, the mice were acclimatized to a room temperature of $22^{\circ} \mathrm{C}$. While adjusting the light/dark cycle to $12 \mathrm{~h} / 12 \mathrm{~h}$, the mice were provided food and water ad libitum for seven days. This study was conducted in accordance with the recommendations of the Ethics Committee of the First Affiliated Hospital of Chongqing Medical University. The protocol was approved by the Ethics Committee of the First Affiliated Hospital of Chongqing Medical University. The number is Lot 2016-34.

2.2. CLP-Induced Model. CLP was performed under anesthesia to determine the position of the cecum. A 3-0 silk suture at $75 \%$ of the distance from the tip was made with a 21 -gauge needle. Sham-operated control mice were subjected to the same surgical laparotomy; the cecum was exteriorized and manipulated as described, but was not ligated or punctured. After $24 \mathrm{~h}$, the experimental mice in the sham operation or CLP operation were sacrificed at the same time, and their lung tissues, blood samples, and spleens were collected for analysis. After ligating the blood vessels leading to the lung and left bronchus in the mice, we injected $200 \mu \mathrm{L}$ phosphate-buffered saline (PBS) into the right lung of the mice through the trachea. After $10 \mathrm{~s}$, we took out the PBS we injected and collected bronchoalveolar lavage fluid (BALF). After two washes, the collected BALF reached 400 cubic feet. Then, we analyzed the collected serum, BALF, spleen, and lung tissues.
2.3. Treatment with Luteolin. To clarify the therapeutic effects of luteolin, the mice were intraperitoneally injected with 10\% DMSO diluted in PBS (control and CLP groups) or $0.2 \mathrm{mg} / \mathrm{kg}$ luteolin (RHAWN ${ }^{\mathrm{TM}}$, China) (CLP+Lut group) $1 \mathrm{~h}$ after CLP stimulation to assess the role of luteolin in ALI/ARDS. The dosage of luteolin was described in previous studies $[14,15]$. Blood, BALF, spleen, and lung tissues were collected at predetermined time points.

2.4. Blockade of Tregs In Vivo. The anti-mouse CD25/IL-2R $\alpha$ antibody (AF2438, R\&D Systems, Minnesota, USA) was used to inhibit the production of Tregs in mice. Anti-CD25 antibody (AF2438, R\&D Systems) or control IgG1 was administered at a dose of $20 \mu \mathrm{g} /$ mouse $1 \mathrm{~h}$ prior to initiating CLP [16].

2.5. Histopathology. The upper lobe of the right lung was treated with $10 \%$ formalin PBS and, after fixation for $24 \mathrm{~h}$, was dehydrated across an ethanol gradient and embedded with paraffin wax. Paraffin sections were observed by hematoxylin and eosin (H\&E) staining. Lung injury scores were estimated by the Mikawa method [17], which comprises the following four categories of lung injury: alveolar congestion, hemorrhage, gap or vascular wall neutrophil infiltration or aggregation, and alveolar septal thickening or transparent membrane formation, and the score interval of each area was $0-4$. No or very slight damage was 0 partitions, mild injury was 1 compartment, moderate injury was between 2 partitions, serious injury was a 3-point interval, and very serious damage was between 4 partitions. As the pathological score of ALI, the higher the total score of accumulated lesions, the more serious the injury.

2.6. Western Blot Analysis. Lung tissues were lysed and homogenized in RIPA buffer in the presence of a protease inhibitor and phosphatase inhibitor; nucleoproteins were extracted after cytosolic proteins were isolated. Protein concentrations were determined using a BCA Assay kit (Beyotime, China) according to the manufacturer's instructions. Equal amounts of proteins were separated using SDSPAGE. The proteins were then transferred to polyvinylidene fluoride (PVDF) membranes, then incubated with antiphospho-p65 (1:500 dilution), anti-p65 (1:500 dilution), anti-FOXP3 (1:500 dilution), or anti-histone H3 (1:1,000 dilution) at $4^{\circ} \mathrm{C}$ overnight. Densitometric analysis of bands was performed using an imaging system (Bio-Rad). All experiments were repeated thrice using different samples.

2.7. Immunohistochemistry. After dewaxing the lung slices with xylene, the lung sections were rehydrated with an alcohol gradient, and then $3 \% \mathrm{H}_{2} \mathrm{O}_{2}$ was used to block endogenous peroxidase activity for $10 \mathrm{~min}$. Fifteen minutes later, $0.3 \%$ Triton X-100 (50 ul) was added to permeabilize the cells. Then, the slices were incubated in normal goat serum. In a humid environment at $4^{\circ} \mathrm{C}$, polyclonal goat anti-mouse IL-17A or polyclonal goat anti-MPO (diluted at $15 \mu \mathrm{g} / \mathrm{mL}$ ) was added to the section and incubated overnight. Diaminobenzidine (DAB) was used to visualize IL-17A or MPO, while hematoxylin was used to stain the nuclei. Images were 
captured using a LEICA CTR 5500 microscope and analyzed using Image-Pro Plus 6.0 software.

2.8. Immunofluorescence. $0.3 \%$ Triton $\mathrm{X}-100(50 \mathrm{ul})$ was added to the frozen lung slices recovered by PBS, and the slices were permeabilized for $15 \mathrm{~min}$. After that, antigen retrieval was performed with sodium citrate solution, and then the slices were incubated in normal goat serum. Polyclonal rabbit anti-mouse $\mathrm{F} 4 / 80$, polyclonal rabbit antimouse iNOS, and polyclonal rabbit anti-mouse CD206 were added to the slices in a humidified chamber at $4^{\circ} \mathrm{C}$ and incubated overnight. Sections were subsequently kept at room temperature for $1 \mathrm{~h}$. Then, at normal room temperature, goat anti-rabbit PE-Cy3 (F4/80), goat anti-rabbit Alexa Fluor (iNOS), and goat anti-rabbit FITC (CD206) were used to stain the section, and nuclei was stained with $4^{\prime}, 6$-diamino2-benzoindole (DAPI) for $1 \mathrm{~h}$. Then, the slices were sealed with neutral glue. Five positive cells at 400x magnification were captured and analyzed. The reagents used in this study were purchased from Servicebio, Wuhan, China.

2.9. Mouse Serum and BALF Cytokine Measurements. Serum and BALF were frozen at $-80^{\circ} \mathrm{C}$ until the analysis. TNF- $\alpha$ IL1, IL-6, IL-10, and IL-17A expression levels were determined using a mouse cytokine/chemokine magnetic bead panel kit (eBioscience, San Diego, CA, USA).

2.10. PBMCs and Splenic Mononuclear Cell Isolation. PBMCs were isolated from mouse heparinized whole blood using a mouse mononuclear cell isolation kit (TBD Science, Tianjin, China) according to the manufacturer's instructions. The splenic mononuclear cells were isolated by density centrifugation as previously described [18]. Briefly, splenic mononuclear cells were isolated from splenic cell suspensions using Ficoll-Paque density gradient (GE Healthcare, USA). The frequency of $\mathrm{CD} 4^{+} \mathrm{CD} 25^{+} \mathrm{FOXP} 3^{+}$Tregs was determined by flow cytometry.

2.11. Flow Cytometry. PBMCs and splenic mononuclear cell isolation were isolated from recipient mice and stained for markers using anti-CD4-FITC, anti-CD25-PE, and antiFOXP3-APC. For intracellular FOXP3 analysis, cells were fixed and permeated using a fixation/permeabilization kit (eBioscience) according to the manufacturer's instructions. At least $10^{5}$ cells were collected with a CytoFLEX flow cytometer (Beckman Coulter, CA, USA) and were analyzed with FlowJo software V10.

2.12. Treg Differentiation. Naïve $\mathrm{CD} 4^{+} \mathrm{T}$ cells were sorted from wild-type C57BL/6 mouse spleens using an EasySep ${ }^{\mathrm{TM}}$ Mouse Naïve $\mathrm{CD}^{+}{ }^{+} \mathrm{T}$ Cell Isolation Kit (STEMCELL). About 500,000 cells were plated in 48 -well plates in $0.5 \mathrm{~mL}$ of complete RPMI 1640 medium (Gibco) supplemented with $10 \%$ FBS (Gibco) and stimulated with anti-mouse CD3 $(5 \mu \mathrm{g} / \mathrm{mL}$, eBioscience) and anti-mouse CD28 $(2 \mu \mathrm{g} / \mathrm{mL}$, eBioscience $)$ plus IL-2 (10 ng/mL, PeproTech), TGF- $\beta 1$ ( $50 \mathrm{ng} / \mathrm{mL}$, PeproTech), $2 \mathrm{mM}$ L-glutamine (STEMCELL), and $50 \mathrm{mM} \beta$-mercaptoethanol (Macklin) in the absence or presence of $2 \mu \mathrm{M}$ luteolin $\left(\mathrm{RHAWN}^{\mathrm{TM}}\right)$ for $4 \mathrm{~d}$ at $37^{\circ} \mathrm{C}$ with $5 \% \mathrm{CO}_{2}$. Finally,
CD4-FITC, CD25-PE, and FOXP3-APC were used to analyze the cells.

2.13. RAW 264.7 Macrophage Culturing and Treatment. RAW 264.7 macrophages were purchased from the American Type Culture Collection (ATCC, Rockville, MD, USA) and cultured in Dulbecco's modified Eagle's medium (DMEM, Gibco) supplemented with 10\% heat-inactivated fetal bovine serum (Gibco). The cells were incubated in a humidified atmosphere at $37^{\circ} \mathrm{C}$ with $5 \% \mathrm{CO}_{2}$. The cells were divided into three groups as follows: cells incubated with culture medium containing $0.1 \%$ DMSO (control group), cells stimulated with LPS (30 ng/mL; Sigma-Aldrich) (LPS group), and cells stimulated with LPS (30 ng/mL; Sigma-Aldrich) plus IL-10 (100 ng/mL; R\&D Systems) (LPS+IL-10 group). After $24 \mathrm{~h}$, the membrane surface molecules were stained with $3 \mu \mathrm{L} /$ test of $\mathrm{PE}$-conjugated monoclonal anti-mouse CD86 (Invitrogen) or APC-conjugated monoclonal antimouse CD206 (Invitrogen) for $30 \mathrm{~min}$ at room temperature in the dark according to the manufacturer's instructions.

2.14. Statistical Analysis. The analysis of all data is mainly conducted by GraphPad Prism 6.01 (GraphPad software, San Diego, CA, USA) and SPSS 19.0 (IBM, Armonk, NY, USA). All statistical data were expressed as the mean \pm standard deviation (SD). To evaluate differences between groups, Student's $t$ test, Mann-Whitney $U$ test, one- or two-way ANOVA test, and Kruskal-Wallis test were used as appropriate. One-way ANOVA was directly conducted using the least significant difference (LSD) multiple comparison test, Dunn test, or Bonferroni multiple comparison test after special testing. Differences were considered statistically significant at $p<0.05$.

\section{Results}

3.1. Luteolin Inhibits Inflammation and Exerts Therapeutic Effects on ALI. Mice (five per group) were sacrificed $24 \mathrm{~h}$ after CLP. Inflammatory tissue damage in the lungs was assessed by histological observation. The results were consistent between experiments; the most representative experimental results are shown in Figure 1. CLP significantly increased lung injury. Treatment with luteolin significantly reduced the extent of lung injury. Comparison of scores of lung injury such as alveolar wall thickening, alveolar hemorrhage, alveolar collapse, and inflammatory cell infiltration in the CLP group revealed that lung injury in the CLP+Lut group was alleviated by luteolin $(p<0.01$, Figure $1(\mathrm{~b}))$, as shown by the lung injury score. After treatment with luteolin, serum concentrations of cytokines IL- $1 \beta$, IL- 6 , TNF- $\alpha$, and IL17A significantly decreased. Moreover, the inflammatory mediators in BALF, including IL- $1 \beta$, IL- 6 , and TNF- $\alpha$, were also markedly reduced (Figure 1(a)). Serum and BALF IL10 levels were significantly increased after luteolin treatment (Figure 2(d)).

3.2. Effects of Luteolin on the Regulation of IL-17A, Neutrophils, and NF- $\kappa B$ p65 in the Lungs of CLP Mice. After treatment with luteolin, pulmonary IL-17A protein levels decreased $(p<0.0001)$, and the proportion of $\mathrm{MPO}^{+}$ 

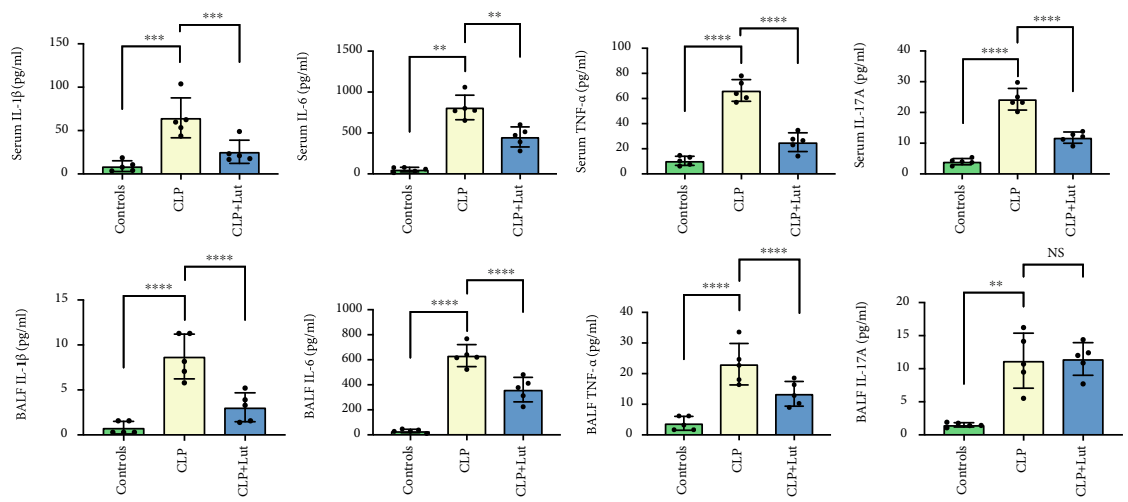

(a)
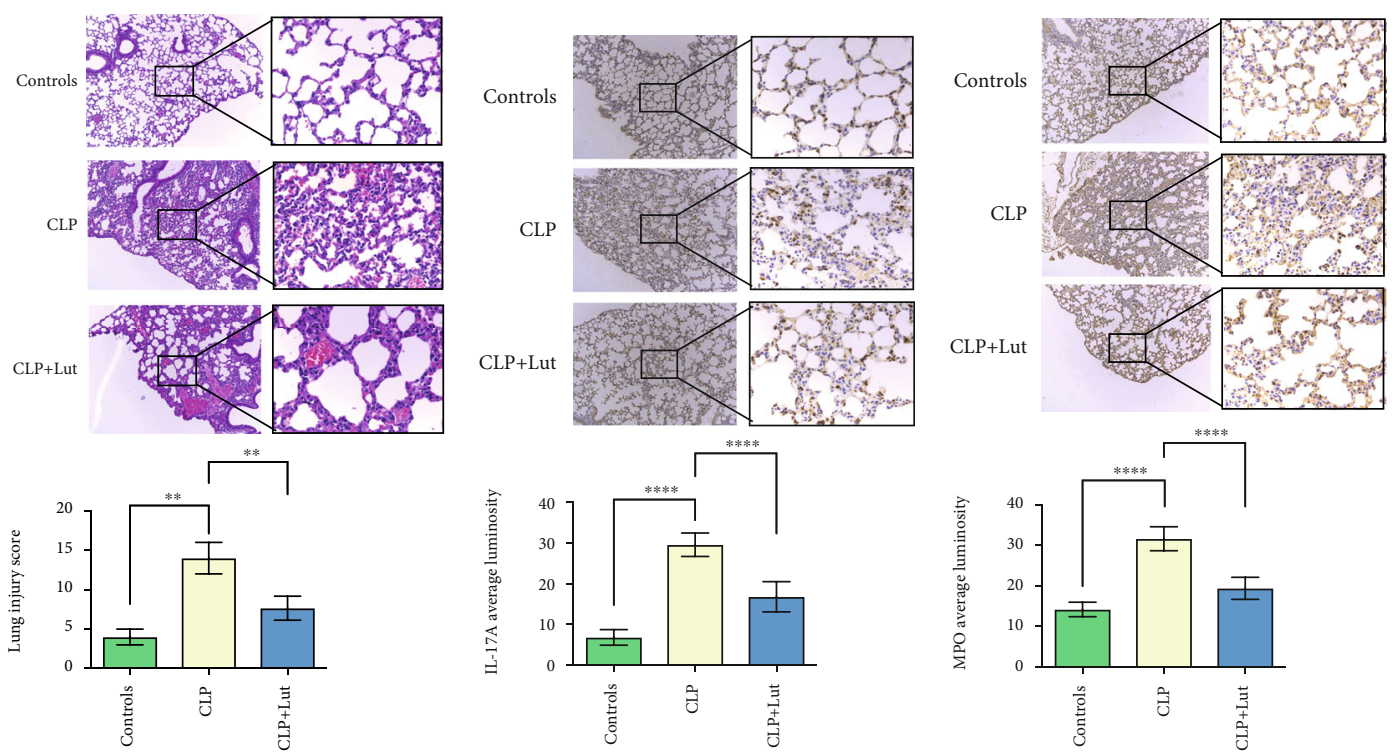

(b)

(c)

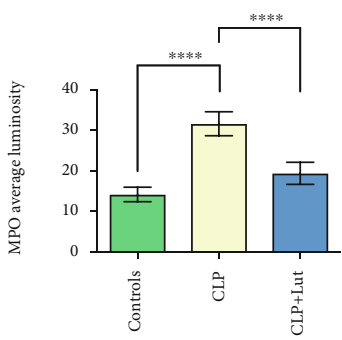

(d)

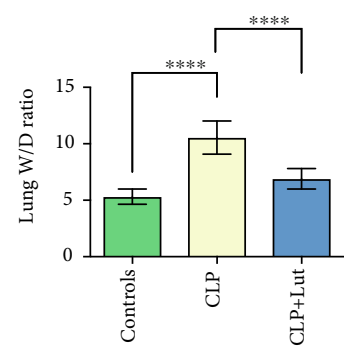

(e)
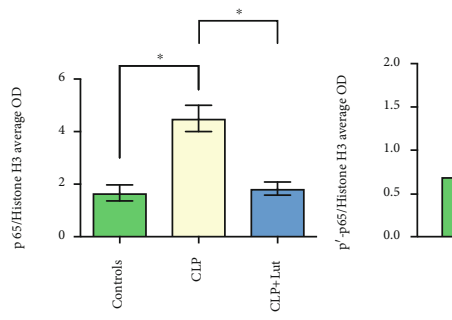

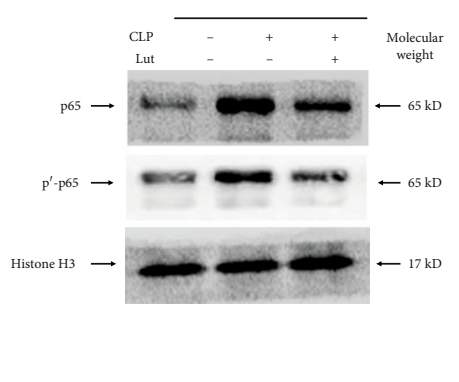

(f)

FIgURE 1: Treatment with luteolin reduces the severity of ALI. (a) Treatment with luteolin reduces the production of IL-1 $\beta$, IL-6, IL-17A, and TNF- $\alpha$ in serum and BALF of CLP mice, thereby playing an anti-inflammatory role. IL-1 $\beta$, IL-6, IL-17A, and TNF- $\alpha$ levels in serum and BALF of mice were measured using a mouse cytokine/chemokine magnetic bead panel kit. (b) Luteolin treatment alleviates lung injury in CLP-induced ALI mice. Lungs from each experimental group were stained with H\&E and processed for histological examination. CLP-induced ALI mice exhibited obvious lung injury. The CLP+Lut group exhibited a significant reduction in the thickness of the alveolar wall, alveolar hemorrhage and collapse, and inflammatory cell infiltration relative to the CLP group. Lung injury in the CLP+Lut group was milder than that in the CLP group. (c, d) Luteolin reduced the proportion of MPO producing neutrophils and IL-17A protein levels in the lungs of CLP mice, thereby playing a protective role in ALI. Immunohistochemical staining for MPO and IL-17A was performed on paraffin-embedded, formalin-fixed lung tissue slices as described in Materials and Methods. (e) Luteolin alleviates pulmonary edema in the CLP-induced mouse model. Pulmonary edema was measured by the lung $W / D$ weight ratio. (f) Luteolin reduced the nuclear translocation of NF- $\kappa \mathrm{B}$ (p65) and NF- $\kappa$ B (p65) phosphorylation activation in lungs of CLP mice. p65 protein levels were measured by western blotting. Each group $n=5$, experiments are repeatable, and the most representative one was shown. Data of the column graphs are presented as means \pm SD. NS: not significant. ${ }^{*} p<0.05,{ }^{* *} p<0.01,{ }^{* * *} p<0.001$, and ${ }^{* * * *} p<0.0001$ by the one-way ANOVA followed by LSD multiple comparison test, compared between the control, CLP, and CLP+Lut groups. 

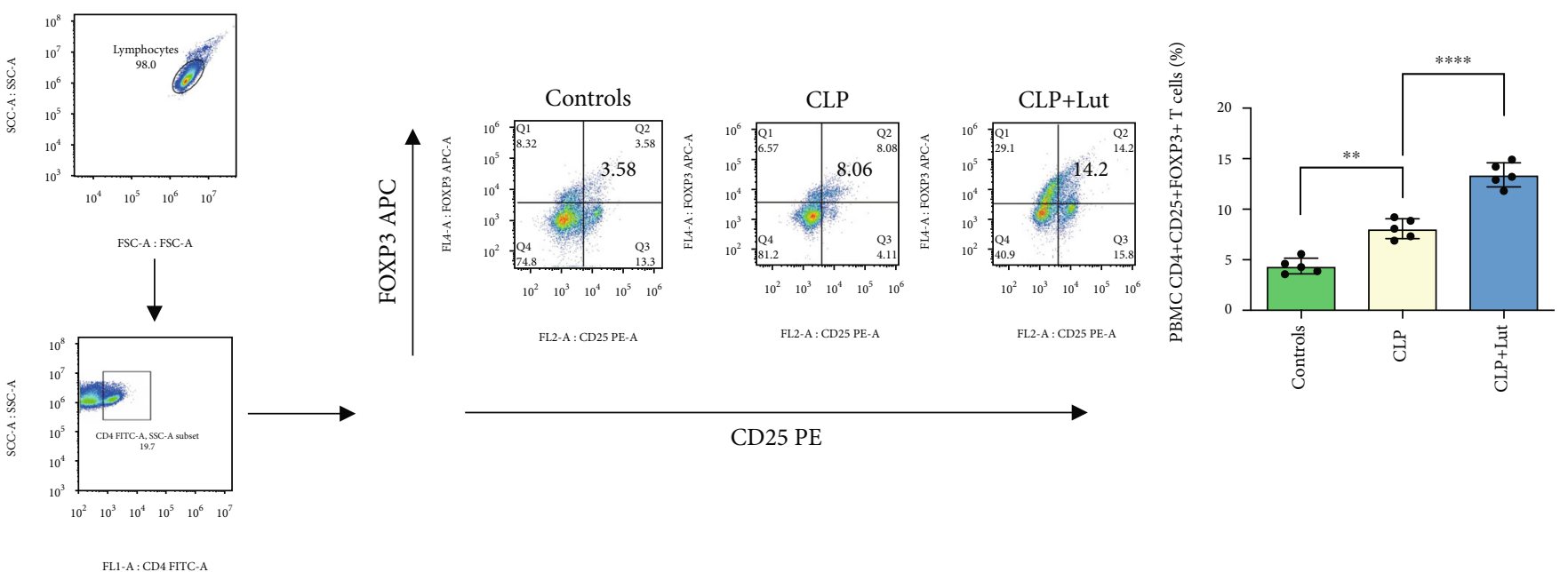

(a)
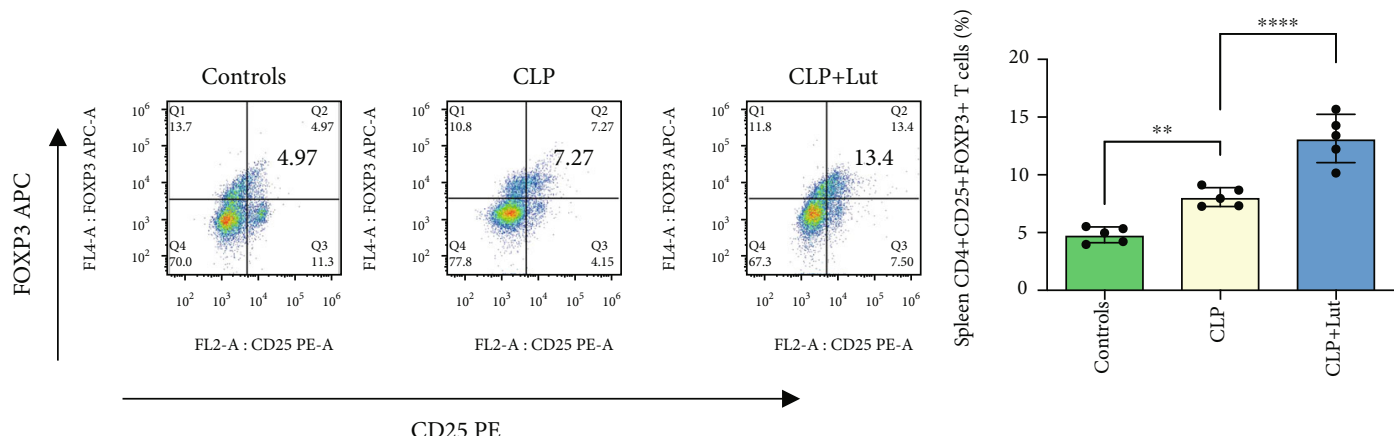

(b)
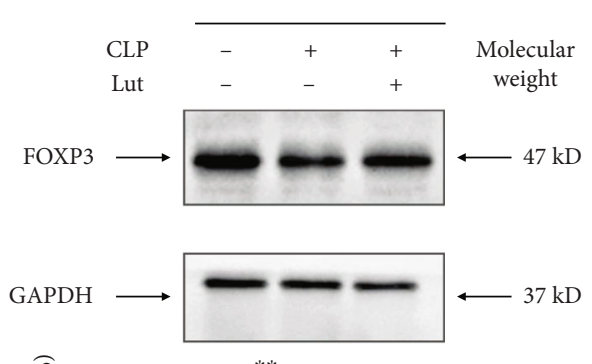

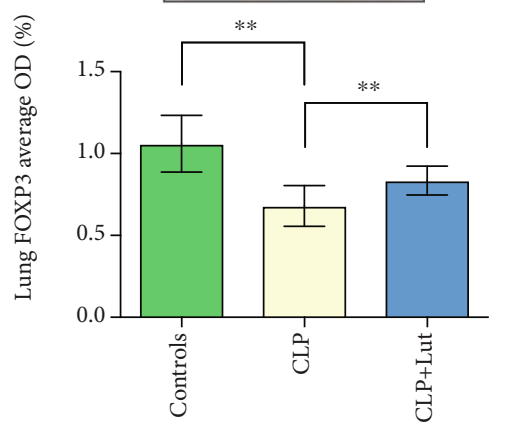

(c)
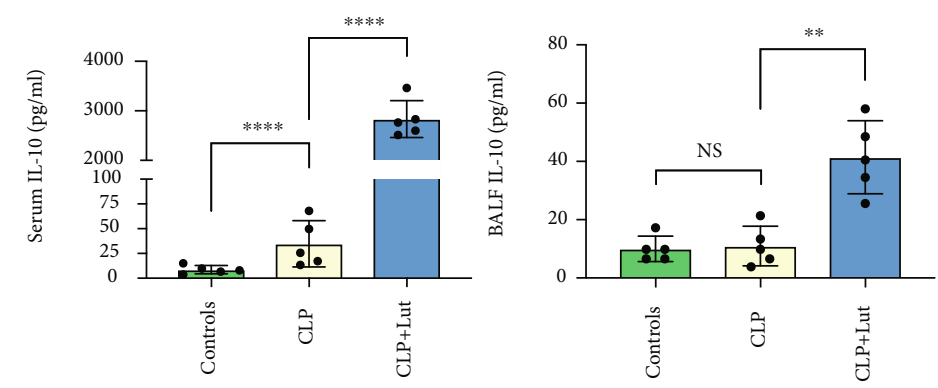

(d)

FIgURE 2: Treatment with luteolin increases the proportion of Tregs and the expression of Foxp3 in the CLP-induced mouse model. (a, b) Treatment with luteolin significantly increased the proportion of $\mathrm{CD} 4{ }^{+} \mathrm{CD} 25^{+} \mathrm{FOXP}{ }^{+}$Tregs in splenic mononuclear cells and PBMCs of CLP mice. $\mathrm{CD} 4^{+} \mathrm{CD} 25^{+} \mathrm{FOXP}^{+}$Tregs in splenic mononuclear cells and PBMCs were detected by flow cytometry. (c) Treatment with luteolin increased the expression of Foxp3 in lungs of CLP mice. Foxp3 protein levels were measured by western blot. (d) Treatment with luteolin promotes the expression of IL-10 in serum and BALF of CLP mice, thereby playing an anti-inflammatory role. IL-10 levels in serum and BALF of mice were measured using a mouse cytokine/chemokine magnetic bead panel kit. Each group $n=5$, experiments are repeatable, and the most representative one was shown. Data of the column graphs are presented as means \pm SD. NS: not significant. ${ }^{*} p<0.05,{ }^{* *} p<0.01,{ }^{* * *} p<0.001$, and ${ }^{* * * *} p<0.0001$ by the one-way ANOVA followed by LSD multiple comparison test, compared between the control, CLP, and CLP+Lut groups. 
neutrophils in the lungs was also reduced $(p<0.001)$ compared with that of the CLP mice (Figures 1(c) and 1(d)). We extracted nuclear proteins from mouse lung tissues to determine the effect of luteolin on NF- $\kappa \mathrm{B}$ (p65) nuclear translocation and NF- $\kappa \mathrm{B}$ (p65) phosphorylation by western blotting. Compared with the control group, the nuclear translocation of NF- $\kappa \mathrm{B}$ (p65) significantly increased after CLP. Meanwhile, the expression level of phosphorylatedp65 (p-p65) in lung tissues of the CLP mice also increased (Figure 1(f)). To assess the degree of lung edema, the $W / D$ ratio was calculated by dividing the wet weight by the dry weight. Compared with the control, the lung wet/dry weight ratios significantly increased after CLP. After luteolin treatment, lung $W / D$ weight ratios were reduced $(p<0.0001)$ compared with CLP mice (Figure 1(e)).

3.3. Luteolin Treatment Increases the Proportion of $\mathrm{CD} 4^{+} \mathrm{CD} 25^{+} \mathrm{FOXP}^{+}$Tregs and the Expression of FOXP3 in the CLP-Induced Mouse Model. The proportion of $\mathrm{CD}^{+-}$ $\mathrm{CD} 25^{+} \mathrm{FOXP}^{+}$Tregs in splenic mononuclear cells and in PBMCs was detected by flow cytometry. We found luteolin treatment significantly increased the proportion of Tregs in the CLP-induced mouse model (Figures 2(a) and 2(b)). FOXP3 is expressed preferentially in Tregs, and lack of FOXP3 protein leads to lack of functional Tregs. After luteolin treatment, western blot analysis showed that the expression of FOXP3 in lung tissues was upregulated compared with the CLP group (Figure 2(c)).

3.4. M1 and M2 Macrophage Phenotypes in the Lungs Are Regulated by Luteolin. Mouse lungs were stained by immunohistochemistry and imaged by fluorescence microscopy. The regulatory levels of luteolin on M1 and M2 macrophages in lung tissues were mainly analyzed by triple immunofluorescence (red: F4/80, pink: NOS $^{+} \mathrm{M} 1$, and green: $\mathrm{CD}^{206}{ }^{+}$ $\mathrm{M} 2$ ). Five fields were chosen from each immunohistochemical section; each field was analyzed by microscope (400x). Compared with the CLP group, we found that the number of M1 cells labeled with pink fluorescence after luteolin treatment significantly decreased $(p<0.0001)$, while the number of M2 cells labeled with green fluorescence significantly increased $(p<0.0001)$ (Figure 3).

3.5. Protective Effects of Luteolin in ALI Are Related to Tregs and $I L-10$. To further explore the role of Tregs in CLPinduced lung injury, CD25 neutralizing antibodies were used to deplete the Tregs after luteolin treatment. The results showed that the proportion of Tregs in splenic mononuclear cells and the level of IL-10 in serum decreased after CD25 neutralization. Additionally, lung injury was aggravated. These results suggest that luteolin targets Tregs to regulate IL-10 levels (Figures 4(a), 4(c), and 4(d)). Moreover, after CD25 was neutralized, western blotting showed that the expression of FOXP3 in the lungs decreased compared with that of the CLP+Lut group (Figure 4(b)).

3.6. Luteolin Promotes the Differentiation of Naïve $C D 4^{+} T$ Cells into Tregs In Vitro, and IL-10 Improves the Polarization of M1/M2 Macrophages. In vitro, we tested whether Treg differentiation and regulation of IL-10 secretion were influenced by luteolin. Naïve $\mathrm{CD}^{+} \mathrm{T}$ cells were isolated from the mice. Then, these cells were divided into two groups: the control and luteolin groups. The results showed that the proportion of $\mathrm{CD} 4{ }^{+} \mathrm{CD} 25^{+} \mathrm{FOXP}^{+}$Tregs was significantly higher after treatment with luteolin (Figure 5(a)). In addition, our in vitro results show that the anti-inflammatory cytokine IL-10 can promote the polarization of M2 macrophages, while the polarization of M1 macrophages continuously decreased (Figure 5(b)).

\section{Discussion}

ARDS/ALI is a severe diffuse lung disease and is a common cause of death in the intensive care unit [19]. The main feature of ALI is the rupture of the alveolar-capillary interface, the secretion of proinflammatory cytokines, and the infiltration of proinflammatory cells [20]. The regulation of uncontrolled inflammation is important in the development of new treatment strategies for developing lung injury. Tregs regulate the activity of $\mathrm{CD}^{+}, \mathrm{CD}^{+} \mathrm{T}$ cells, $\mathrm{B}$ cells, natural killer cells, dendritic cells, and other immune cells mainly through contact-dependent inhibition or release inhibition of cytokines IL-10 and TGF- $\beta$ and can play an anti-inflammatory role at this time [21]. Tregs play an important role in relieving inflammation and promoting lung repair in ARDS [22, 23]. The mortality of ARDS is closely related to the increase of the $\mathrm{CD}^{+} \mathrm{CD} 25^{+} \mathrm{FOXP} 3^{+}$Treg ratio in the alveoli [24]. Tregs are considered as potential therapeutic targets for ARDS mainly because they play an important role in alleviating inflammation.

Luteolin is a flavone compound present in many medicinal plants. It not only has anti-inflammatory effects but also has various biological and pharmacological effects [7]. Recent studies have reported that luteolin suppresses the expression of proinflammatory cytokines, including IL-17, IL-1 $\beta$, TNF$\alpha$, and IL- 6 , which are key modulators of both acute and chronic inflammation [25]. Luteolin attenuates the effects of sepsis-induced ALI by suppressing the ICAM-1, iNOS pathways, oxidative stress, and NF- $\kappa \mathrm{B}$ [15]. In the current study, most research choice pretreatment methods. However, there are an increasing number of experts who think that pretreatments are clinically meaningless. A study comparing different ARDS/ALI models showed that CLP treatment also results in the early elevation of cytokine and plasma TNF- $\alpha$ and IL- 6 levels $2 \mathrm{~h}$ after CLP, and animal body temperature markedly decrease between 2 and $12 \mathrm{~h}$ [26]. This is an indicator that systemic inflammation has developed. The development of ARDS/ALI is closely related to uncontrolled inflammation. Treatment within two hours after CLP may aid in developing better therapeutic strategies for the treatment of proinflammatory disorders. In our research, mice in the CLP+Lut group exhibited less severe lung tissue damage compared with those in the CLP group (Figure 1(b)). Protein levels of IL-17A and MPO, which could injure the alveolar epithelium and induce neutrophil aggregation in lung tissue, were significantly reduced (Figures 1(c) and $1(\mathrm{~d}))$. The NF- $\kappa \mathrm{B}$ p65 subunit is phosphorylated and translocated into the nucleus to regulate the transcription of proinflammatory mediators [27]. The NF- $\kappa$ B p 65 phosphorylation 
Control
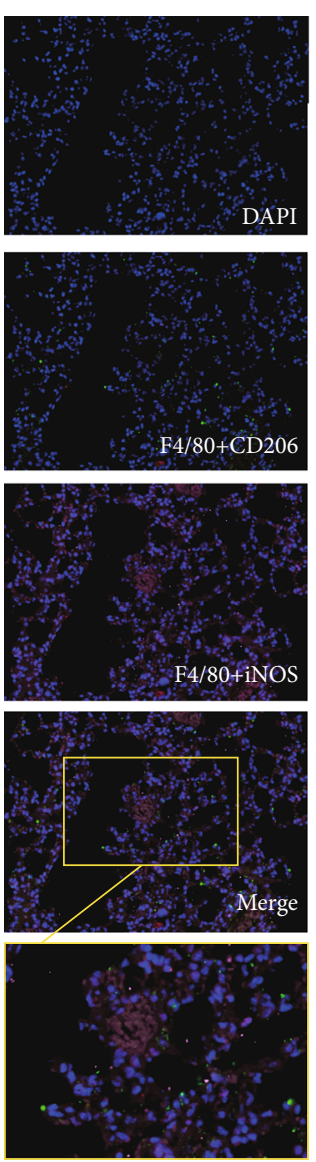

CLP
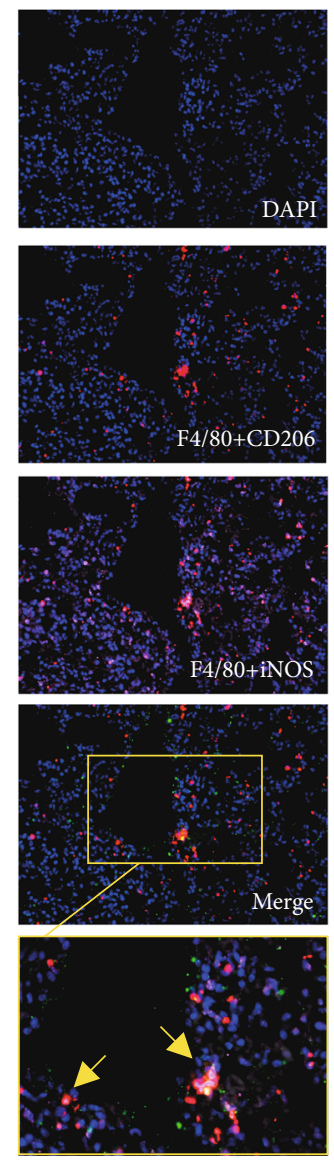

(a)
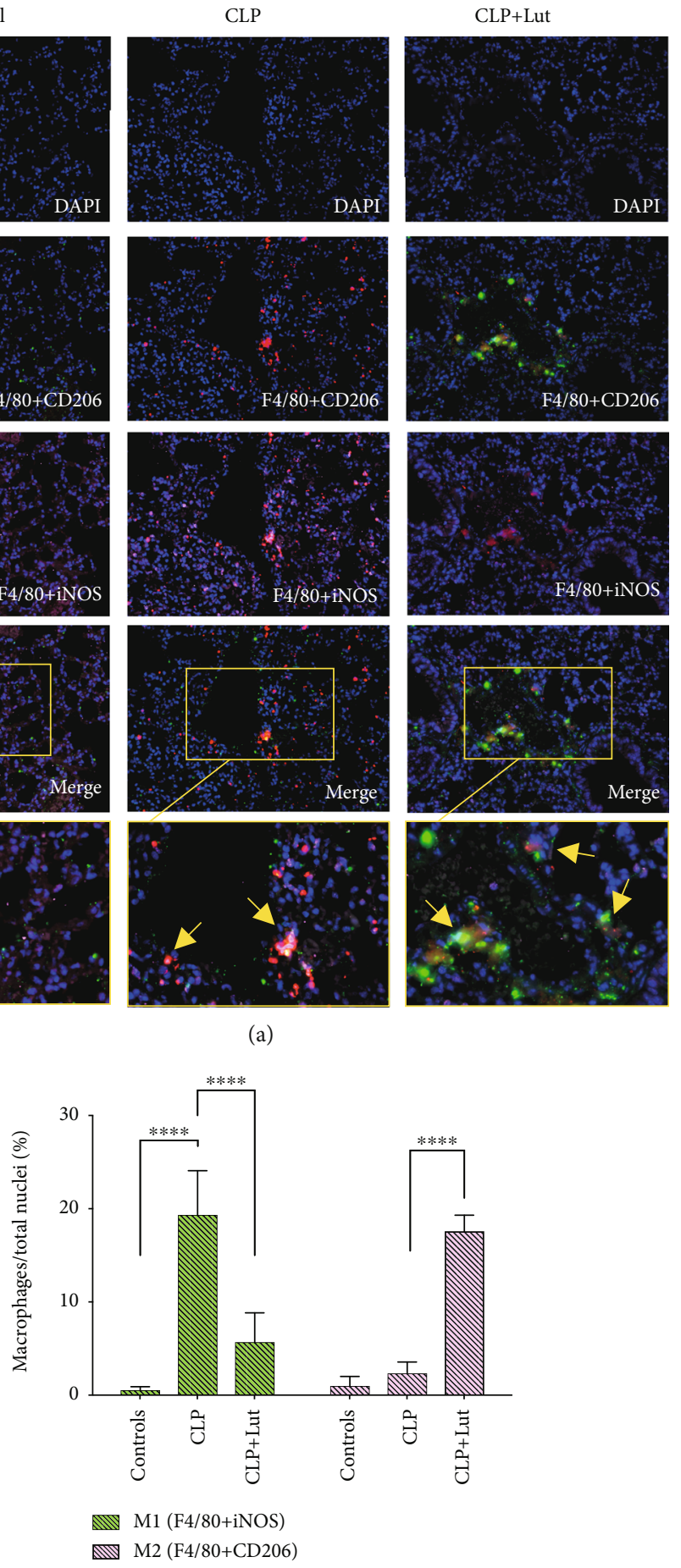

(b)

FIgURE 3: Treatment with luteolin regulates M1 and M2 macrophage phenotypes in lungs of CLP mice. (a) Treatment with luteolin reduces the proportion of M1 macrophages, while increasing the proportion of M2 macrophages in lungs of CLP mice, hence playing a protective role in ALI. The proportion of pink fluorescent iNOS expressing M1 macrophages increased significantly, while lungs of CLP mice were substantially negative for green fluorescent $\mathrm{CD} 206^{+} \mathrm{M} 2$ macrophages. Treatment with luteolin reduced the proportion of M1 while increasing that of M2 macrophages in ALI. Five 400x fields of positive cells were counted and analyzed. Each group $n=5$, experiments are repeatable, and the most representative one was shown. Data of the column graphs are presented as means $\pm \mathrm{SD}$. ${ }^{* * * *} p<0.0001$ by the one-way ANOVA followed by LSD multiple comparison test, compared between the control, CLP, and CLP+Lut groups.

activation and nuclear translocation of NF- $\kappa$ B (p65) significantly increased after CLP. After luteolin treatment, NF- $\kappa \mathrm{B}$ p65 phosphorylation activation and nuclear translocation of
NF- $\kappa \mathrm{B}$ (p65) decreased compared with those of the CLP group (Figure 1(f)). In ARDS, immune cells secrete cytokines and chemokines to initiate and maintain the uncontrolled 

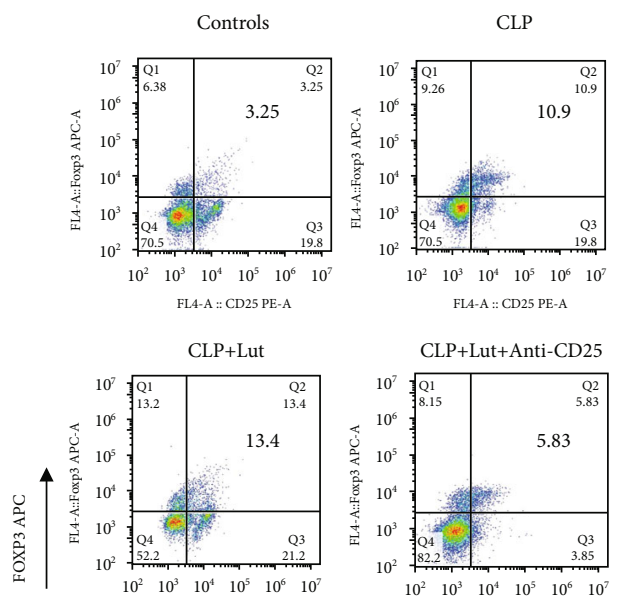

CLP+Lut+Anti-CD25

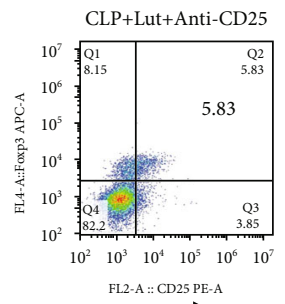

CD25 PE
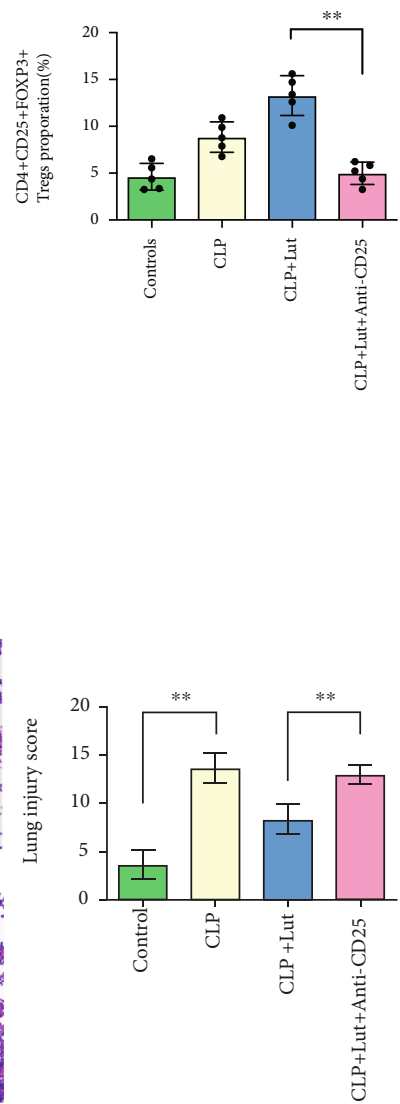

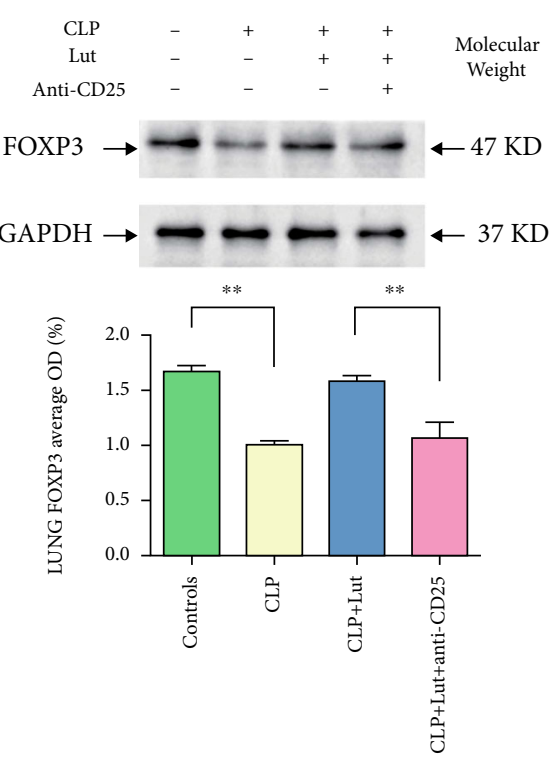

(b)

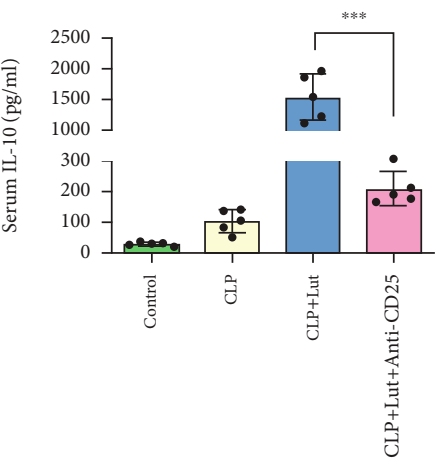

(d)

FIgURE 4: Luteolin plays a protective role in ALI, which is potentially linked to IL-10 and Tregs. (a, c, d) After treatment with CD25 neutralizing antibodies, the number of Tregs in the spleen of mice in the CLP+Lut group was significantly reduced. IL-10 expression was significantly reduced, and lung injury was significantly worse. $\mathrm{CD} 4{ }^{+} \mathrm{CD} 25^{+} \mathrm{FOXP} 3^{+}$Tregs in splenic mononuclear cells were detected by flow cytometry. Lungs from each experimental group were stained with H\&E and processed for histological examination. Mouse IL-10 serum levels were measured by ELISA. (b) After treatment with CD25 neutralizing antibodies, the expression of Foxp3 in lungs of GL+CLP mice was decreased. Foxp3 protein levels were measured by western blot. Each group $n=5$, experiments are repeatable, and the most representative one was shown. Data of the column graphs are presented as means \pm SD. ${ }^{* *} p<0.01$ and ${ }^{* * *} p<0.001$ between groups represented by horizontal lines, as calculated by one-way ANOVA followed by LSD multiple comparison test.

inflammatory response. The levels of proinflammatory cytokines TNF- $\alpha$, IL- $1 \beta$, IL- 6 , and IL-17A in mouse serum significantly decreased after treatment with luteolin. Moreover, the inflammatory mediators in BALF, including IL- $1 \beta$, IL-6, and TNF- $\alpha$, were also markedly reduced (Figure 1(a)). In our study, we found that after treatment with luteolin, the concentrations of IL-17A significantly decreased in the mouse serum at $24 \mathrm{~h}$, but did not decrease in mouse BALF. In the process of lung injury, IL-17A expression exhibited differences at various time points; this may be because we used a CLP-induced ALI mouse model. In this model, sepsis originated from a polymicrobial infectious focus within the abdominal cavity, followed by bacterial translocation into the blood compartment, which then triggers a lung inflammatory response [28]. The expression of IL-17A in the mouse serum immediately decreased after inflammation was blocked, but in BALF, this may be delayed. As a consequence, luteolin reduced the degree of lung injury because it can inhibit the production or function of inflammatory mediators.

Currently, information of the mechanisms by which luteolin regulates Tregs is limited. Previous studies have shown that luteolin affects asthma via the induction of $\mathrm{FOXP}^{+}$and $\mathrm{CD} 4^{+} \mathrm{CD} 25^{+}$Tregs or acts as an immunosuppressant $\mathrm{mTOR}$ inhibitor to increase the percentage of $\mathrm{CD} 4^{+-}$ FOXP3 $^{+}$Tregs posttransplantation [29]. The main results show that (i) the inhibition function of $\mathrm{CD}^{+} \mathrm{FOXP}^{+}$Tregs is enhanced or the proliferation speed of Treg in vivo is promoted after activation or (ii) the signal pathway regulated by immunoregulatory cytokines, small molecule inhibitors, or therapeutic antibodies is stimulated [30]. In our study, we observed that the Treg ratio in splenic mononuclear cells and PBMCs of mice significantly increased (Figure 2(a) and 2(b)). 

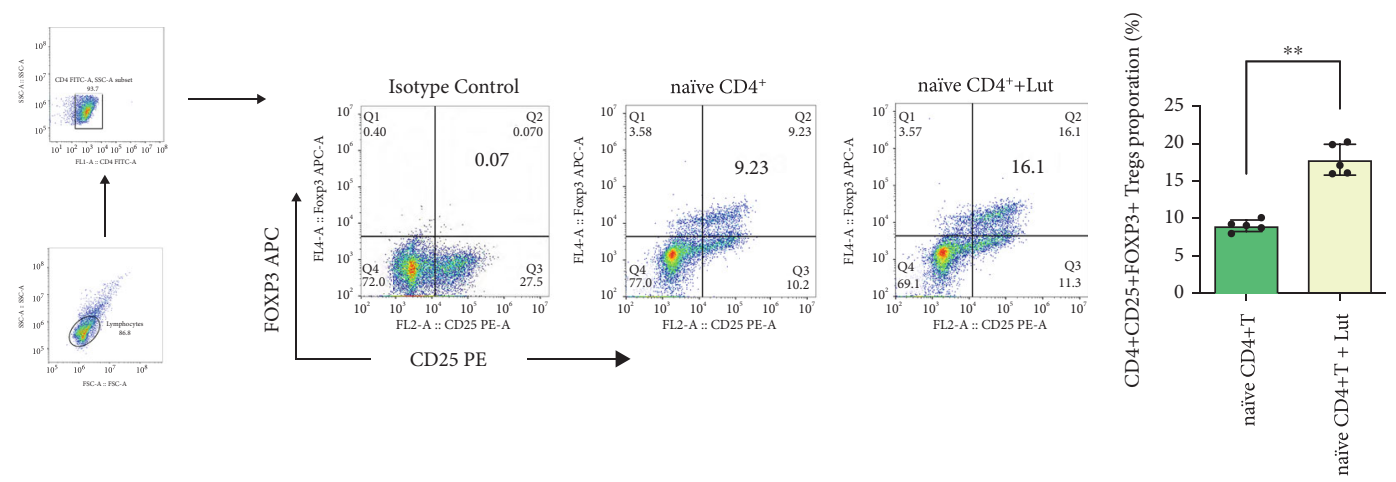

(a)
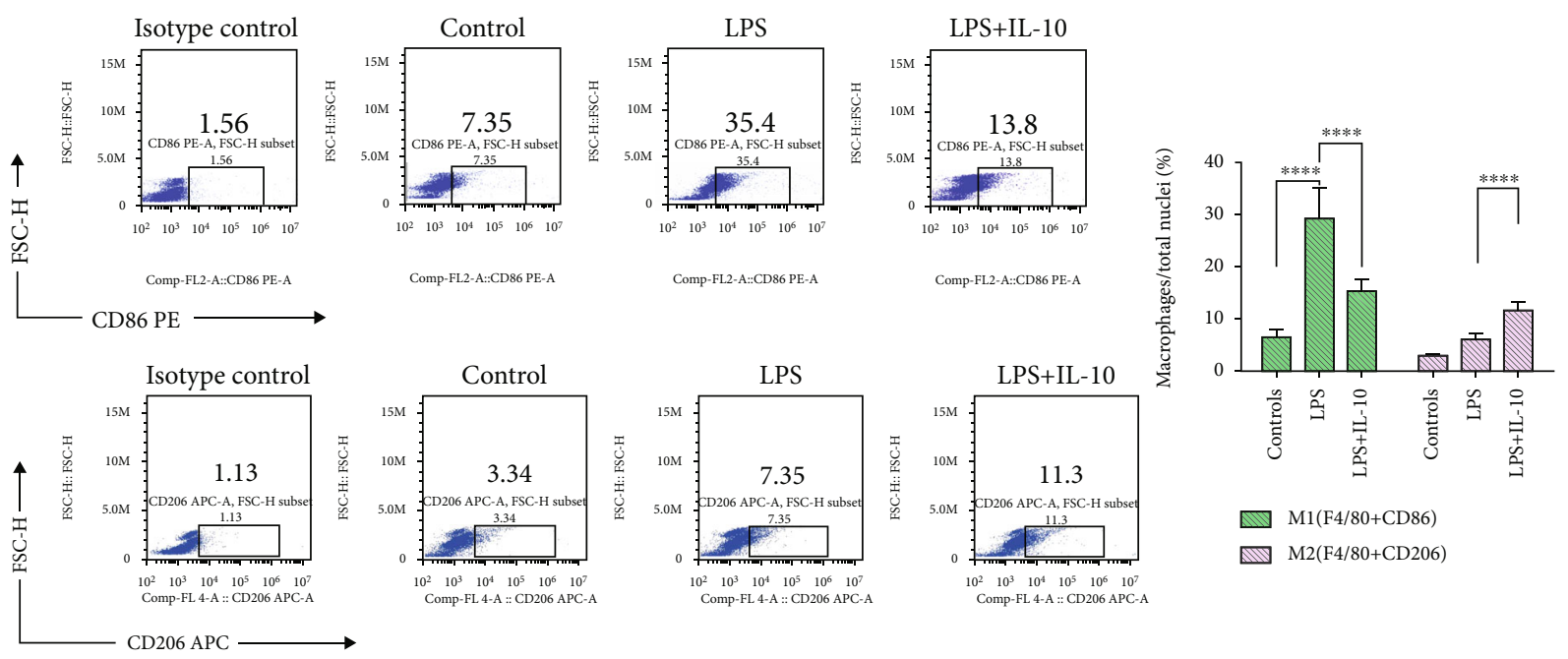

(b)

Figure 5: Luteolin promotes the differentiation of naïve $\mathrm{CD}^{+} \mathrm{T}$ cells into Tregs, and IL-10 improves the polarization of M1/M2 macrophages. (a) Luteolin significantly promotes the differentiation of naïve CD4 ${ }^{+} \mathrm{T}$ cells into $\mathrm{CD} 4^{+} \mathrm{CD} 25^{+} \mathrm{FOXP} 3^{+} \mathrm{Tregs}^{\mathrm{Naïve}} \mathrm{CD} 4^{+} \mathrm{T}$ cells were sorted from the spleen of wild-type C57BL/6 mice and cultured as described in Materials and Methods. Tregs were detected by flow cytometry on day 4. (b) IL-10 affects the polarization of M1/M2 macrophages. Proportions of M1/M2 macrophages were examined by flow cytometry on day 3. Each group $n=5$, experiments are repeatable, and the most representative one was shown. Data of the column graphs are presented as means \pm SD. ${ }^{* *} p<0.01$ and ${ }^{* * * *} p<0.0001$ comparing the control, LPS, and LPS+IL-10 groups, as calculated by one-way ANOVA followed by the LSD multiple comparison test.

CD25, the alpha chain of IL-2 receptor (IL-2R), is expressed in an inducible manner and required for formation of the high affinity IL-2R [31]. CD25 is absent or minimally expressed on resting $\mathrm{T}$ and NK cells, but its transcription is potently induced on T cells stimulated via the TCR or IL-2. After T cell activation, CD25 is rapidly induced and high-affinity receptors form [32]. CD25 is an important surface marker for Tregs and is often used to purify and sort Tregs. CD4, CD25, and FOXP3 triple marker-positive is a relative standard method to identify Tregs [33]. Tregs constitutively express CD25, and CD4 ${ }^{+-}$ $\mathrm{CD} 25^{+}$Tregs suppress $\mathrm{CD} 4^{+} \mathrm{CD} 25^{-} \mathrm{T}$ cells in a cell contactdependent manner in vitro [34]. In our research, the total number of $\mathrm{CD}^{2} 5^{+} \mathrm{FOXP}^{-}$cells in the flow cytometry plots increased in the CLP+Lut group compared with the CLP group (Figures 2(a) and 2(b)), indicating that luteolin could promote Treg differentiation in ARDS. FOXP3 is specifically in the Tregs expressed and controls their development and function. Western blotting showed that after treatment with luteolin, the expression of FOXP3 in the lungs was also upregulated compared with the CLP group (Figure 2(c)), indicating that luteolin promotes Treg differentiation and the expression of FOXP3 in ARDS. Another study showed that the decrease in FOXP3 expression in patients is due to the decrease of FOXP3 expression in $\mathrm{CD}^{+}{ }^{+} \mathrm{T}$ cells, which leads to Treg dysfunction in patients [35]. Our study shows that luteolin treatment results in the upregulation of FOXP3 in the lungs compared with CLP mice, which may be related to the increase in lung Tregs and its protective effects. However, the underlying mechanism requires further investigation. Moreover, comparison of the CLP+Lut group with the CLP group revealed that the levels of Treg-related cytokines IL-2 and IL-10 in the CLP+Lut group significantly increased (Figure 2(d)), thus providing preliminary evidence that luteolin regulates Tregs and thereby improves the inflammatory response and reduces lung injury. Simultaneously, Tregs originate from naïve $\mathrm{CD}^{+} \mathrm{T}$ cells. A variety of effector $\mathrm{T}$ cell lineages, including Tregs, is formed by the differentiation of naive $\mathrm{CD} 4^{+} \mathrm{T}$ cells. A recent study showed that the inflammatory microenvironment is coupled 
with metabolism and can induce phenotypic remodeling of $\mathrm{CD}^{+} \mathrm{T}$ cells [36]. Thus, it is essential to determine the effects of luteolin. In our in vitro study, after stimulation with luteolin, the proportion of $\mathrm{CD} 4^{+} \mathrm{CD} 25^{+} \mathrm{FOXP}^{+}$Tregs significantly increased (Figure 5(a)). These results indicate that one of the mechanisms by which luteolin regulates Tregs may be by enhancing the differentiation of naïve $\mathrm{CD}^{+} \mathrm{T}$ cells into Tregs.

Because Tregs can act as a "cytokine sink" for different effector cytokines [4], the effects of luteolin on Tregs might be mainly mediated by cytokines. One critical suppressive mechanism of Tregs is the secretion of inhibitory cytokines such as IL-10 and TGF- $\beta$ [37]. IL-10 is an antiinflammatory cytokine and protects against lung injury. In our study, the protective effect of luteolin in ALI is closely related to Tregs and IL-10. The increased expression of IL-10 is also due to luteolin treatment (Figure 2(d)). Luteolin and Tregs have a certain synergistic effect in regulating the expression of IL-10 in cells. However, the protective effect of IL-10 in ALI not only inhibits the production of proinflammatory cytokines but also mediates the activity of neutrophils [38] and dendritic cells [39]. Importantly, IL-10 is a key signaling molecule for macrophages in their response to inflammation [40]. The anti-inflammatory effects of IL-10 are mediated by metabolic reprogramming of macrophages, including inhibition of mTOR signaling and inflammasome activation [11]. Macrophages play an important role in ARDS when they encounter an inflammatory reaction. Macrophages have both proinflammatory and anti-inflammatory effects, which change with the microenvironment of different pathological stages [41]. In terms of macrophage phenotypes in the mouse lung, we found that the M1 phenotype was more prevalent in the CLP group than in the CLP+Lut group, and the opposite was observed for M2 macrophages. Luteolin treatment significantly decreased the proportion of M1 macrophages and increased the proportion of M2 macrophages (Figure 3), which coincides with changes in IL-10 levels in plasma and Tregs of splenic mononuclear cells and PBMCs upon luteolin treatment. A similar observation was reported in a previous study; Il-10 derived from Tregs is one of the main factors affecting macrophage polarization [42]. Therefore, the present study explored the interaction between Tregs and other effector cells (such as macrophages) and the influence of cytokines secreted by Tregs (such as IL-10) on this interaction. Another research also observed that at postinfection, IL-10, an anti-inflammatory cytokine produced by Tregs and antagonizes Th1 cytokines, was also highly increased [43]. In addition, our in vitro experiments further proved that IL-10 could induce macrophage polarization toward the M2 phenotype and suppress macrophage polarization toward the M1 phenotype in vitro (Figure 5(b)). In lung injury, M1 macrophages play the role of promoter; on the contrary, the level of proinflammatory cytokines in ARDS is limited by M2 macrophage and participates in the process of lung tissue repair. Our experiment suggested that luteolin exerts its anti-inflammatory effects and is closely related to regulating Treg differentiation, promoting IL-10 expression, and then interfering with macrophage polarization.
In sum, luteolin imparts a therapeutic effect on ARDS, which includes the regulation of Tregs and reduction in proinflammatory cytokine production. These therapeutic effects could indirectly affect other immune cells (e.g., macrophages) through cytokines secreted by Tregs. Our findings show that luteolin promotes Treg cell differentiation and increases the production of IL-10, which may interfere with the polarization of macrophages.

\section{Conclusions}

Luteolin alleviates lung injury and suppresses uncontrolled inflammation by inducing the differentiation of $\mathrm{CD}^{+-}$ $\mathrm{CD}_{25}{ }^{+} \mathrm{FOXP}^{+}{ }^{+}$Tregs and upregulating the expression of IL-10. Furthermore, the anti-inflammatory cytokine IL-10 can promote polarization of M2 macrophages in vitro. Luteolin induces the differentiation of Tregs from naïve $\mathrm{CD} 4^{+} \mathrm{T}$ cells and may be a potential mechanism for regulating IL-10 production.

\section{Data Availability}

All data generated or analyzed during this study are included in this article.

\section{Conflicts of Interest}

The authors declare no conflict of interest.

\section{Authors' Contributions}

Conception, hypothesis, and design were handled by Fang $\mathrm{Xu}$ and Chuan-jiang Wang. Data acquisition and analysis were handled by Ke Xie, Yu-sen Chai, Chuan-jiang Wang, and Fang $\mathrm{Xu}$. Manuscript preparation was handled by $\mathrm{Ke}$ Xie and Fang Xu. Literature search was handled by Shi-hui Lin, Yu-sen Chai, and Fang Xu.

\section{Acknowledgments}

This study was supported by the Basic Science and CuttingEdge Technology Research Projects of Chongqing Science and Technology Commission (cstc2020jcyj-msxmX0014 to CJ-W), the Chinese Medicine Science and Technology Project of Chongqing City Health and Family Planning Committee (2020GDRC001 to FX) and High-Level Medical Reserved Personnel Training Project of Chongqing (to FX).

\section{References}

[1] M. A. Matthay, L. B. Ware, and G. A. Zimmerman, "The acute respiratory distress syndrome," The Journal of Clinical Investigation, vol. 122, no. 8, pp. 2731-2740, 2012.

[2] G. Rawal, S. Yadav, and R. Kumar, "Acute respiratory distress syndrome: an update and review," Journal of Translational Internal Medicine, vol. 6, no. 2, pp. 74-77, 2018.

[3] C. Y. Yang, C. S. Chen, G. T. Yiang et al., "New insights into the immune molecular regulation of the pathogenesis of acute respiratory distress syndrome," International Journal of Molecular Sciences, vol. 19, no. 2, p. 588, 2018. 
[4] S. Lin, H. Wu, C. Wang, Z. Xiao, and F. Xu, "Regulatory T cells and acute lung injury: cytokines, uncontrolled inflammation, and therapeutic implications," Frontiers in Immunology, vol. 9, p. 1545, 2018.

[5] P. L. Silva, P. Pelosi, and P. R. M. Rocco, "Personalized pharmacological therapy for ARDS: a light at the end of the tunnel," Expert Opinion on Investigational Drugs, vol. 29, no. 1, pp. 49-61, 2020.

[6] Z. Jia, P. Nallasamy, D. Liu et al., "Luteolin protects against vascular inflammation in mice and TNF-alpha-induced monocyte adhesion to endothelial cells via suppressing $\mathrm{I} K \mathrm{~B} \alpha / \mathrm{NF}-\kappa \mathrm{B}$ signaling pathway," The Journal of Nutritional Biochemistry, vol. 26, no. 3, pp. 293-302, 2015.

[7] N. Aziz, M. Y. Kim, and J. Y. Cho, "Anti-inflammatory effects of luteolin: a review of in vitro, in vivo, and in silico studies," Journal of Ethnopharmacology, vol. 225, pp. 342-358, 2018.

[8] D. Chen, A. Bi, X. Dong et al., "Luteolin exhibits antiinflammatory effects by blocking the activity of heat shock protein 90 in macrophages," Biochemical and Biophysical Research Communications, vol. 443, no. 1, pp. 326-332, 2014.

[9] C. Nunes, L. Almeida, R. M. Barbosa, and J. Laranjinha, "Luteolin suppresses the JAK/STAT pathway in a cellular model of intestinal inflammation," Food \& Function, vol. 8, no. 1, pp. 387-396, 2017.

[10] E. J. Park, Y. M. Kim, H. J. Kim, and K. C. Chang, "Luteolin activates ERK1/2- and Ca2+-dependent HO-1 induction that reduces LPS-induced HMGB1, iNOS/NO, and COX-2 expression in RAW264.7 cells and mitigates acute lung injury of endotoxin mice," Inflammation Research, vol. 67, no. 5, pp. 445-453, 2018.

[11] W. K. E. Ip, N. Hoshi, D. S. Shouval, S. Snapper, and R. Medzhitov, "Anti-inflammatory effect of IL-10 mediated by metabolic reprogramming of macrophages," Science, vol. 356, no. 6337, pp. 513-519, 2017.

[12] K. Minton, "IL-10 targets macrophage metabolism," Nature Reviews. Immunology, vol. 17, no. 6, p. 345, 2017.

[13] S. H. Kim, E. Saba, B. K. Kim et al., "Luteolin attenuates airway inflammation by inducing the transition of $\mathrm{CD} 4^{+} \mathrm{CD} 25^{-}$to $\mathrm{CD} 4^{+} \mathrm{CD} 25^{+}$regulatory T cells," European Journal of Pharmacology, vol. 820, pp. 53-64, 2018.

[14] A. Kotanidou, A. Xagorari, E. Bagli et al., "Luteolin reduces lipopolysaccharide-induced lethal toxicity and expression of proinflammatory molecules in mice," American Journal of Respiratory and Critical Care Medicine, vol. 165, no. 6, pp. 818-823, 2002.

[15] S. Rungsung, T. U. Singh, D. J. Rabha et al., "Luteolin attenuates acute lung injury in experimental mouse model of sepsis," Cytokine, vol. 110, pp. 333-343, 2018.

[16] L. Zhou, C. Chu, F. Teng et al., "Innate lymphoid cells support regulatory $\mathrm{T}$ cells in the intestine through interleukin-2," Nature, vol. 568, no. 7752, pp. 405-409, 2019.

[17] K. Mikawa, K. Nishina, Y. Takao, and H. Obara, "ONO-1714, a nitric oxide synthase inhibitor, attenuates endotoxininduced acute lung injury in rabbits," Anesthesia and Analgesia, vol. 97, no. 6, pp. 1751-1755, 2003.

[18] M. Tian, Y. Zhang, Z. Liu, G. Sun, G. Mor, and A. Liao, “The PD-1/PD-L1 inhibitory pathway is altered in pre-eclampsia and regulates T cell responses in pre-eclamptic rats," Scientific Reports, vol. 6, no. 1, p. 27683, 2016.

[19] X. Liu, S. Jiang, Q. Zhang et al., “Tim-3 regulates Tregs' ability to resolve the inflammation and proliferation of acute lung injury by modulating macrophages polarization," Shock, vol. 50, no. 4, pp. 455-464, 2018.

[20] G. W. Tu, Y. Shi, Y. J. Zheng et al., "Glucocorticoid attenuates acute lung injury through induction of type 2 macrophage," Journal of Translational Medicine, vol. 15, no. 1, p. 181, 2017.

[21] F. Zhang, M. Y. Li, Y. T. Lan, and C. B. Wang, "Imbalance of Th17/Tregs in rats with smoke inhalation-induced acute lung injury," Scientific Reports, vol. 6, no. 1, p. 21348, 2016.

[22] B. D. Singer, J. R. Mock, N. R. Aggarwal et al., "Regulatory T cell DNA methyltransferase inhibition accelerates resolution of lung inflammation," American Journal of Respiratory Cell and Molecular Biology, vol. 52, no. 5, pp. 641-652, 2015.

[23] N. Terada, H. Kuroda, H. Nakayama, K. Matsumoto, and Y. Kitamura, "Effect of genetically defined oocyte depletion on production of androgens and oestrogens by ovaries of suckling mice," Journal of Steroid Biochemistry, vol. 25, no. 1, pp. 83-89, 1986.

[24] M. Adamzik, J. Broll, J. Steinmann et al., "An increased alveolar CD4 + CD25 + Foxp3 + T-regulatory cell ratio in acute respiratory distress syndrome is associated with increased 30-day mortality," Intensive Care Medicine, vol. 39, no. 10, pp. 1743-1751, 2013.

[25] M. D. Turner, B. Nedjai, T. Hurst, and D. J. Pennington, "Cytokines and chemokines: at the crossroads of cell signalling and inflammatory disease," Biochimica et Biophysica Acta, vol. 1843, no. 11, pp. 2563-2582, 2014.

[26] S. Seemann, F. Zohles, and A. Lupp, "Comprehensive comparison of three different animal models for systemic inflammation," Journal of Biomedical Science, vol. 24, no. 1, p. 60, 2017.

[27] Y. Li, Q. Gong, W. Guo et al., "Farrerol relieve lipopolysaccharide (LPS)-induced mastitis by inhibiting AKT/NF- $\kappa \mathrm{B}$ p65, ERK1/2 and P38 signaling pathway," International Journal of Molecular Sciences, vol. 19, no. 6, p. 1770, 2018.

[28] D. Rittirsch, M. S. Huber-Lang, M. A. Flierl, and P. A. Ward, "Immunodesign of experimental sepsis by cecal ligation and puncture," Nature Protocols, vol. 4, no. 1, pp. 31-36, 2009.

[29] S. Ye, H. Liu, Y. Chen et al., "A novel immunosuppressant, luteolin, modulates alloimmunity and suppresses murine allograft rejection," Journal of Immunology, vol. 203, no. 12, pp. 3436-3446, 2019.

[30] Z. Chen, F. Lin, Y. Gao et al., "FOXP3 and ROR $\gamma$ t: transcriptional regulation of Treg and Th17," International Immunopharmacology, vol. 11, no. 5, pp. 536-542, 2011.

[31] K. Fu, X. Sun, W. Zheng et al., "Sam68 modulates the promoter specificity of NF- $\kappa \mathrm{B}$ and mediates expression of CD25 in activated T cells," Nature Communications, vol. 4, no. 1, p. 4, 2013.

[32] W. Liao, J. X. Lin, and W. J. Leonard, "Interleukin-2 at the crossroads of effector responses, tolerance, and immunotherapy," Immunity, vol. 38, no. 1, pp. 13-25, 2013.

[33] L. M. R. Ferreira, Y. D. Muller, J. A. Bluestone, and Q. Tang, "Next-generation regulatory T cell therapy," Nature Reviews. Drug Discovery, vol. 18, no. 10, pp. 749-769, 2019.

[34] O. Cao, E. Dobrzynski, L. Wang et al., "Induction and role of regulatory $\mathrm{CD} 4+\mathrm{CD} 25+\mathrm{T}$ cells in tolerance to the transgene product following hepatic in vivo gene transfer," Blood, vol. 110, no. 4, pp. 1132-1140, 2007.

[35] M. Xu, Q. Liu, S. Li et al., "Increased expression of miR-338-3p impairs Treg-mediated immunosuppression in pemphigus vulgaris by targeting RUNX1," Experimental Dermatology, vol. 29, no. 7, pp. 623-629, 2020. 
[36] V. Pucino, M. Certo, V. Bulusu et al., "Lactate buildup at the site of chronic inflammation promotes disease by inducing $\mathrm{CD}^{+} \mathrm{T}$ cell metabolic rewiring," Cell Metabolism, vol. 30, no. 6, pp. 1055-1074.e8, 2019, e1058.

[37] J. Zhu, H. Yamane, and W. E. Paul, "Differentiation of effector CD4 T cell populations (*)," Annual Review of Immunology, vol. 28, no. 1, pp. 445-489, 2010.

[38] G. Inoue, "Effect of interleukin-10 (IL-10) on experimental LPS-induced acute lung injury," Journal of Infection and Chemotherapy, vol. 6, no. 1, pp. 51-60, 2000.

[39] R. Kapur, M. Kim, R. Aslam et al., "T regulatory cells and dendritic cells protect against transfusion-related acute lung injury via IL-10," Blood, vol. 129, no. 18, pp. 2557-2569, 2017.

[40] F. Krizan and J. Panduric, "Importance of the palatal seal for complete denture's retention," Acta Stomatologica Croatica, vol. 25, no. 4, pp. 245-251, 1991.

[41] X. Huang, H. Xiu, S. Zhang, and G. Zhang, "The role of macrophages in the pathogenesis of ALI/ARDS," Mediators of Inflammation, vol. 2018, Article ID 1264913, 8 pages, 2018.

[42] Y. S. Chai, Y. Q. Chen, S. H. Lin et al., "Curcumin regulates the differentiation of naive CD4+T cells and activates IL-10 immune modulation against acute lung injury in mice," Biomedicine \& Pharmacotherapy, vol. 125, p. 109946, 2020.

[43] S. Herold, K. Mayer, and J. Lohmeyer, "Acute lung injury: how macrophages orchestrate resolution of inflammation and tissue repair," Frontiers in Immunology, vol. 2, p. 65, 2011. 